\title{
63. GENERATING MECHANISMS FOR SPIRAL WAVES
}

\author{
D. LYNDEN-BELL
}

Royal Greenwich Observatory, Sussex, England

To maintain the spiral structure over long periods in spite of the chaotic disturbances so often seen in galaxies it is probably necessary to have a large energy store which is gradually tapped to generate and maintain the spiral wave. Now in a sense stable axially symmetrical model galaxies are configurations of minimum energy for a given angular momentum structure. By a given angular momentum structure I mean that the function $\mu(h) \mathrm{d} h$ giving the total mass with specific angular momentum between $h$ and $h+\mathrm{d} h$ is given and in making the minimum energy statement $\mathrm{I}$ have assumed that there is sufficient 'random' motion to satisfy Toomre's local stability criterion. To tap a store of energy one must either tap the energy of the whole rotational structure by finding disturbances that may change $\mu(h)$, or one may tap the smaller energy store of the 'random' motions. Clearly the larger energy store is the more attractive. Now $\mu(h)$ is conserved for any axially symmetrical time dependent motions so the only disturbances that can change $\mu(h)$ are non-axially symmetrical. If one considers a single star moving in nearly circular motion and interacting with a weak disturbance in the gravitational potential which may be Fourier analysed into components of the form $S(R) \exp [i(m \phi+\omega t)]$ then one finds that the energy and angular momentum of the star oscillate but do not change in the mean. This statement is untrue when the force due to the wave does not average to zero or when it resonates with the natural oscillation of the star about the circular motion. The angular frequency of the force seen by the star is $\omega+m \Omega$, where $\Omega(R)$ is the rotational angular velocity of the galaxy, so the exceptions occur at the resonances

$$
\omega+m \Omega=0, \pm \kappa
$$

Here $\kappa(R)=(4 B(B-A))^{1 / 2}$, the epicyclic angular frequency, and $A(R)$ and $B(R)$ are Oort's constants. At the radius where $\omega+m \Omega(R)=0$ the pattern of the disturbance moves around at the same velocity as the circular motion of the stars epicentre. The other resonances that were discovered by B. Lindblad lie one within and one outside that circle.

I wish to point out here the strong connection between the physical picture of Landau damping and Landau excitation in the electrostatic waves of an encounterless plasma and the physics of the resonances in the theory of spiral structure. The physical picture of the Landau problem is that a wave that can propagate in the bulk of the plasma will resonate with particles travelling very close to the wave velocity. These special particles must be treated separately. Those that travel just faster than the wave, so little faster that they are unable to climb over the wave crest, together produce a net pushing on the wave in the direction of its propagation through the 
plasma. Conversely those moving just below the wave speed are laggards holding the wave back. Normally there are more laggards than pushers so the wave loses energy and momentum and is damped. If, however, there are more pushers than laggards, as when two streams of particles interpenetrate, the wave is amplified leading to Landau excitation, which is another name for the two stream instability.

Compare this picture with what is happening close to the circle where $\omega+m \Omega(R)=0$. Stars with epicentres just within that circle have mean angular velocity just greater than the wave - they are the pushers; stars with epicentres just outside have mean angular velocity just less than the wave - they are the laggards. If the angular momentum imparted to the wave by the pushers is greater than that extracted from the wave by the laggards then we may expect that the wave will be enhanced. This will clearly happen if the surface density of epicentres falls off rapidly with $R$ close to the resonance. If such a rapid fall occurs at some radius disturbances moving around the galaxy with a pattern speed equal to the circular velocity these will be excited. Outside such a ring we must have an outward going wave since its source will be within. If we write the wave locally in the form $S^{\prime} \exp [i(m \phi+\omega t-k R)]$ then $k / \omega$ must be positive to get an outward moving wave; however $m \Omega$ must have the opposite sign to $\omega$ by the resonance condition. Hence $k / m$ has the same sign as $-\Omega$. Now locally the spiral has the form $\phi=(k / m) R$. Hence $\phi$ increases with $R$ when the picture of the galaxy is drawn with the galactic rotation in the sense of $\phi$ decreasing. In the outer parts such arms will therefore trail. Within the resonant circle the same argument would prove that the wave leads but for the fact that any wave propagating inwards will be reflected in the central parts. Thus within the resonant circle there will be a standing wave with a cartwheel or, in the simplest case, a barred structure. The rather vague outer 'edge' of the galaxy does not likewise reflect a wave for the same reason that a pond with a gradually sloping shore does not reflect water waves. The generating of spiral waves is analogous to the forcing of a semi-infinite stretched string fixed at one end and forced at one point. Between the forcing point and the fixed end a standing wave develops but a propagating wave occurs on the outside.

The resonant circle driving the most prominent spiral structure is not necessarily that with the largest density gradient across it because that circle may be poorly matched to the reflected wave from the centre. However assuming that the matching is never too bad it is interesting to see how the changes in the angular momentum of the stars close to resonance will secularly enhance the generating mechanism. Since stars just inside the resonant circle lose angular momentum they move inwards, while those just outside acquire angular momentum and move outwards. Thus the resonant circle is evacuated with density enhancements on either side. The density enhancement just inside the resonant circle followed by the dearth at the resonant circle gives a very strong gradient of epicentre density just inside the old resonance. We deduce that there is a secular movement of the strongest excitation circle inwards and that it sweeps before it a growing ring of matter. Thus in external galaxies we should see a pronounced ring surrounding a bar structure and the spiral structure should start from the ring. 
I have not investigated the physical mechanisms that may occur at the Lindblad resonances but it is not unlikely that these too can be made to generate spiral structures. However it is not clear to me that these resonances give direct access to the main energy store of the galactic rotation rather than to the smaller energy of epicyclic motion. I remark however that the mechanism I have described seems to lead inevitably to galaxies with some cartwheel, bar-like or ring structure such as de Vaucouleurs's type SABrs and there is obviously room for mechanisms that generate structures devoid of rings or bars. One might speculate that the two main classes of spirals, SB and $\mathrm{S}$ are generated from the resonant circle and from the Lindblad resonance respectively.

None of the statements in this paper have been properly proved by detailed calculations, rather they are a physicist's attempt at a mechanistic explanation. 